\title{
Directions for non-reciprocal electronics
}

\author{
Advanced semiconductor technologies are helping to find new ways to create components capable of one-way \\ transmission.
}

$\mathrm{N}$ on-reciprocal devices are a key component of modern communication technology. Isolators, for example, are two-port non-reciprocal devices that allow a signal to be transmitted only in one direction, and are used to protect wireless transmitters from back reflections from the antenna. Alternatively, circulators are three-port non-reciprocal devices that route a signal from one port to the next only in a clockwise (or counter-clockwise) direction; they can be used to interface a transmitter and a receiver to an antenna, allowing signals to be simultaneously transmitted and received at the same frequency. The components also have a role to play in emerging technologies. They are, for instance, employed in the readout of qubits in superconducting quantum computer, an application in which they must operate at cryogenic temperatures and near the quantum limits of noise.

The devices are typically built using ferrite materials and require large magnetic fields (to exploit the Faraday effect). As a result, they are bulky and expensive, and integrating them in modern semiconductor fabrication processes is problematic. Thus, alternative approaches that do not require magnetic materials and can be integrated on-chip are sought. Attempts to achieve non-magnetic non-reciprocity can be traced back to the $1960 \mathrm{~s}^{1,2}$, but in recent years a variety of new approaches have emerged driven by advances in semiconductor device technology. Aravind Nagulu, Negar Reiskarimian and Harish Krishnaswamy explore these developments in a Review Article in this issue of Nature Electronics.

Non-magnetic non-reciprocal electronics can be built using a variety of methods, including through the use of active transistors and nonlinearity. But the researchers focus on devices based on temporal modulation, which they argue have the greatest potential. They discuss approaches based on the temporal modulation of permittivity or capacitance; this technique has, for example, been used to construct compact circulators via angular momentum biasing of coupled resonant rings ${ }^{3}$. They also discuss approaches based on the temporal modulation of conductivity; this technique has, for example, been used to construct miniaturized circulators that can be integrated into conventional

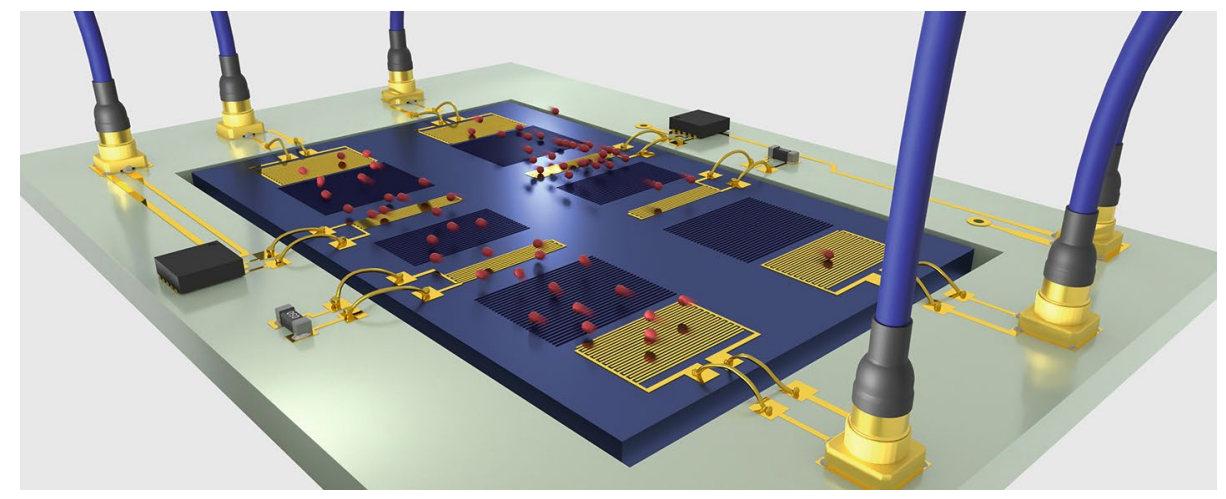

Schematic illustration of a non-reciprocal surface acoustic wave device developed by Shao, Lončar and colleagues ${ }^{7}$. The device, which is built on a lithium niobate platform and contains coupled acoustic resonators, transmits acoustic waves (represented here by red spheres) only in one direction.

Credit: Linbo Shao

complementary metal-oxide-semiconductor (CMOS) technology ${ }^{4}$.

Krishnaswamy and colleagues - who are based Columbia University and Massachusetts Institute of Technology - go on to consider the future challenges for the field, highlighting current limitations in the theoretical analysis of time-modulated circuits, as well as the opportunities offered by topological metamaterials. They also explore the use of superconducting temporally modulated components for quantum computing applications, and the capabilities of hybrid electro-acoustic components.

High-frequency acoustic waves are a valuable information carrier for both classical and quantum applications. But to harness their full benefits, acoustic non-reciprocal devices are required. In an Article elsewhere in this issue, Linbo Shao, Marko Lončar and colleagues at Harvard University, Washington University in St. Louis and the California Institute of Technology show that a nonlinear parity-time symmetric system can achieve nonreciprocal propagation of microwave acoustic waves. Their system (pictured) is based on two coupled acoustic resonators in a lithium niobate platform. Thanks to the strong piezoelectricity of lithium niobate, and with the help of electric circuitry, the gain, loss and nonlinearity of the system can be tuned. And by using two nonreciprocal devices connected in series, the researchers show that one-way circulation of acoustic waves is possible.
The idea of parity-time symmetry first emerged from the study of quantum mechanics ${ }^{5}$, but has since spread to other fields, allowing unique devices to designed. Beyond acoustics, and the work of Shao, Lončar and colleagues, this includes various optical, optomechanical and electronic systems. In a further Article in this issue, Sid Assawaworrarit and Shanhui Fan at Stanford University illustrate what nonlinear parity-time symmetric circuits can do for wireless power transfer.

As we highlighted back in $\mathrm{March}^{6}$, wireless power is of increasing value in a world of mobile devices and electric vehicles. Assawaworrarit and Fan now show that a parity-time symmetric circuit that uses a switch-mode amplifier and current-sensing feedback can provide robust and efficient wireless power transfer. They demonstrate the capabilities of the approach by using it to wirelessly transfer around $10 \mathrm{~W}$ of power to a moving device.

Published online: 22 May 2020 https://doi.org/10.1038/s41928-020-0424-X

References

1. Kamal, A. Proc. IRE 48, 1424-1430 (1960).

2. Tanaka, S., Shimomura, N. \& Ohtake, K. Proc. IEEE 53, 260-267 (1965).

3. Estep, N. A., Sounas, D. L., Soric, J. \& Alù, A. Nat. Phys. 10, 923-927 (2014)

4. Reiskarimian, N. \& Krishnaswamy, H. Nat. Commun. 7, 11217 (2016).

5. Bender, C. M. \& Boettcher, S. Phys. Rev. Lett. 80, 5243 (1998).

6. Nat. Electron. 3, 131 (2020).

7. Shao, L. et al. Nat. Electron. https://doi.org/10.1038/s41928-0200414-z (2020). 